\title{
Calculation of stress and the absorption of Angiotensin in the left ventricle based on two dimensional echocardiograms and MRIs
}

\author{
A. K. Macpherson ${ }^{1}$, S. Neti ${ }^{1}$, M. Averbach ${ }^{2}$ \& P. A. Macpherson ${ }^{3}$ \\ ${ }^{1}$ Institute for Biomedical Engineering and Mathematical Biology, \\ Lehigh University, Bethlehem, PA, USA \\ ${ }^{2}$ Department of Radiology, St Luke's Hospital, Bethlehem, PA, USA \\ ${ }^{3}$ Department of Applied Technology, Rogers State University, Tulsa, OK, \\ USA
}

\begin{abstract}
A cardiologist could make a more accurate diagnosis if the data on the stress and absorption of Angiotensin II in the patient were more readily available. Currently one of the most widely used diagnostic tools is the two dimensional echocardiogram which can generate information to predict levels of stress. An MRI of the heart provides full volumetric coverage of the heart and is more accurate than echocardiograms in calculating left ventricular function. For example in one instance the calculation of the ejection fraction based on echocardiograms was measured to be 65 percent but use of MRI resulted in 79 percent ejection. The calculation of stress must be accomplished quickly so that the information can be integrated with other clinical information to assist with decision making. Presently this requires that the MRI results be limited to two dimensions. The present study compares the boundary conditions for stress calculations obtained from echocardiograms with those using two dimensional MRI data. An obstacle is that the two dimensional slice shown by an echocardiogram is difficult to relate to a slice of an MRI. It is shown that errors in using echocardiograms can be significant at the end of systole when myocardial stresses may be maximal. This is particularly true around the apex where the stress is often considerable. Using the MRI data, results of stress calculations are presented as well as the conditions for the absorption of Ang II. The results are significantly different from those calculated using echocardiograms.
\end{abstract}

Keywords: left ventricle, echocardiograms, MRI, ventricular stress, clinical diagnosis, blood flow. 


\section{Introduction}

The long term objective of the present research is to develop a prediction method for use by clinicians to detect the early stages of the development cardiac hypertrophy. One significant cause of the onset of hypertrophy is mechanical stress (arising from the fluid forces), which is considered to be the trigger inducing a growth response in the overloaded myocardium [1]. Two recent surveys [3, 4] have reached the conclusions that (1) "Cardiac myocytes can sense the stretch induced by stress and convert it into intracellular growth signals" (2) "Mechanical stress is considered to be the trigger inducing a growth response in the overloaded myocardium."

One result of hypertrophy is that gene changes occur in the myocytes cells. To initiate the gene changes within the cells the myocyte stretching requires the presence of the hormone Angiotensin II (Ang II). Ang II produces myocyte enlargement within the left ventricle [3]. Ang II stimulates myocytes by binding to its cognate (G-protein coupled) receptors on myocyte plasmalemma surfaces and initiating signal cascades within the cells leading to an increase in the number of contractile protein units, and thus an increase in sarcomere length.

Thus the provision of data on the regions of left ventricular stress and the corresponding absorption of Angiotensin II of a patient to a cardiologist would be useful in making a diagnosis. However it is becoming evident that there are many interactions involved with hypertrophy other than the direct Ang II-left ventricular interaction. There are Ang II receptors located in cerebrovascular endothelial cells and results in a change in cerebrovascular flow [4].

The calculation of blood flow and the related variation in forces around the ventricle using echocardiograms have been undertaken for a number of years [5-11].

\section{Method of calculation}

In the solution, the blood flow into the left atrium is simulated by a source distributed throughout the atrium. In order to conserve mass, sinks are distributed around the periphery of the integration domain. During systole the aortic valve is modeled as a sink in the ventricular cavity. The change in shape is obtained from the echocardiograms and used as boundary conditions for the flow. The source strength has to match the change in volume of the ventricle. The valves have to be modeled as thicker than in reality as Lagrangian integration must go around both sides of the valve. The Navier-Stokes equations are then solved with a predictor corrector scheme [6].

The Navier-Stokes equations defined on an $\mathrm{x}-\mathrm{y}$ Cartesian co-ordinate system for an incompressible fluid are

$$
\begin{gathered}
\rho\left(\frac{\partial \hat{u}}{\partial t}+\hat{u} \bullet \nabla \hat{u}\right)+\nabla p=\mu \nabla^{2} \hat{u}+\hat{F} \\
\nabla \hat{u}=0
\end{gathered}
$$


where $\hat{u}$ is the velocity vector, $\rho$ is the density, $t$ is the time, $p$ is the pressure and the viscosity is $\mu$.

The boundary force $\hat{F}$ arising from the heart muscles is

$$
\hat{F}(\hat{x}, t)=\int_{0}^{L} \hat{f}(s, t) \delta(\hat{x}-\hat{X}(s, t)) d s
$$

Here $\hat{f}$ is the force on the boundary element at the point $s$ defined on a Lagrangian system where $\hat{x}$ is defined on the Cartesian system and $\hat{X}^{n}$ is the $n t h$ point on the Lagrangian system. The stress is calculated as the gradient of the velocity near the wall. As the shear stress is continuous at the wall interface this will be equal to the shear force on the ventricular wall. The representation of the stress calculations outcome is obviously a function of the accuracy of the motion of the ventricular walls. Thus the present research compares the accuracy of the ventricular wall motion when echocardiograms and MRI of the same patient are compared.

\section{Results}

The echocardiograms are taken at a constant angle with respect to the frame. A possible angle is shown on the MRI cross axis view in fig. 1 . The slices are $8 \mathrm{~mm}$ apart (out of the paper) and are all at the same instance. It can be seen that line intersects the left ventricle in all slices.

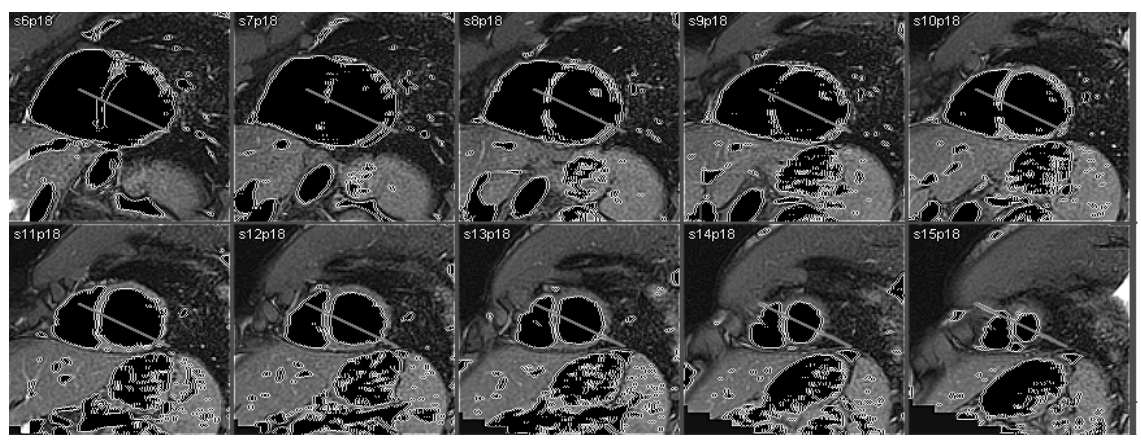

Figure 1: $\quad$ MRI at the beginning of diastole (33 year old male healthy patient).

For this, end of diastole, comparison between the echocardiogram and the MRI myocardium profiles are in good agreement as shown in fig. 2. Such comparisons between MRIs and echocardiograms for the myocardium location are in general good except when we approach the end of systole

As we approach the end of systole the comparison is often difficult or impossible. If the angle at which the echocardiogram is taken is as shown in fig. 3, then the results are as in fig. 3, where the line often misses the ventricle. 
Technicians can and do adjust the direction of the echocardiogram to overcome this difficulty but a comparison of the echocardiogram with an MRI as in fig. 1 becomes impossible.

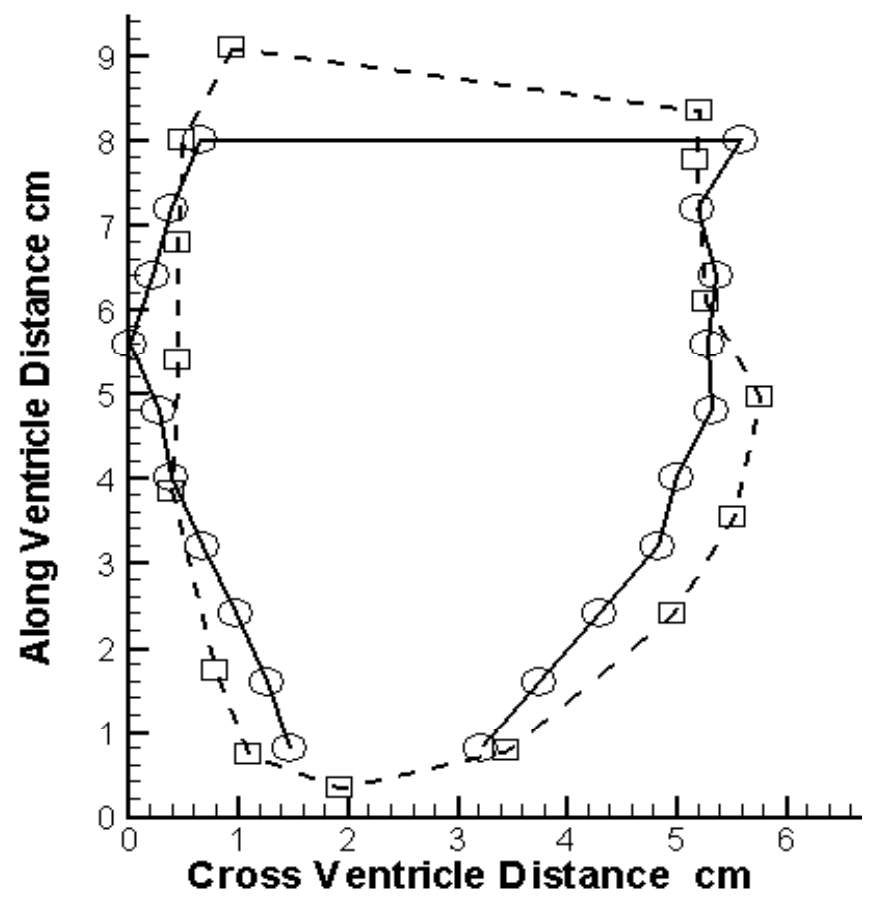

Figure 2: Comparison between echocardiogram and MRI views of left ventricle at the time in fig. 1. Echocardiogram $\square---\square---\square$, MRI ○-০—०.

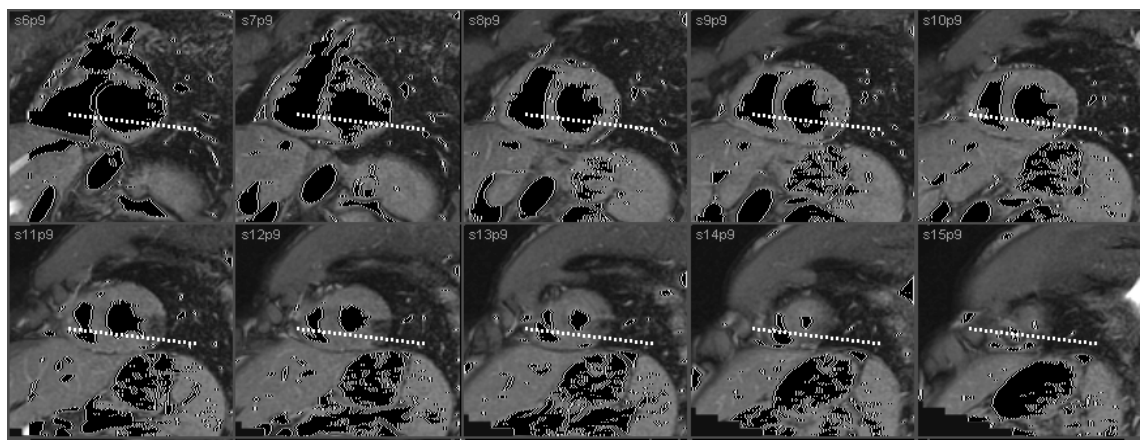

Figure 3: $\quad$ MRI at the end of systole. 


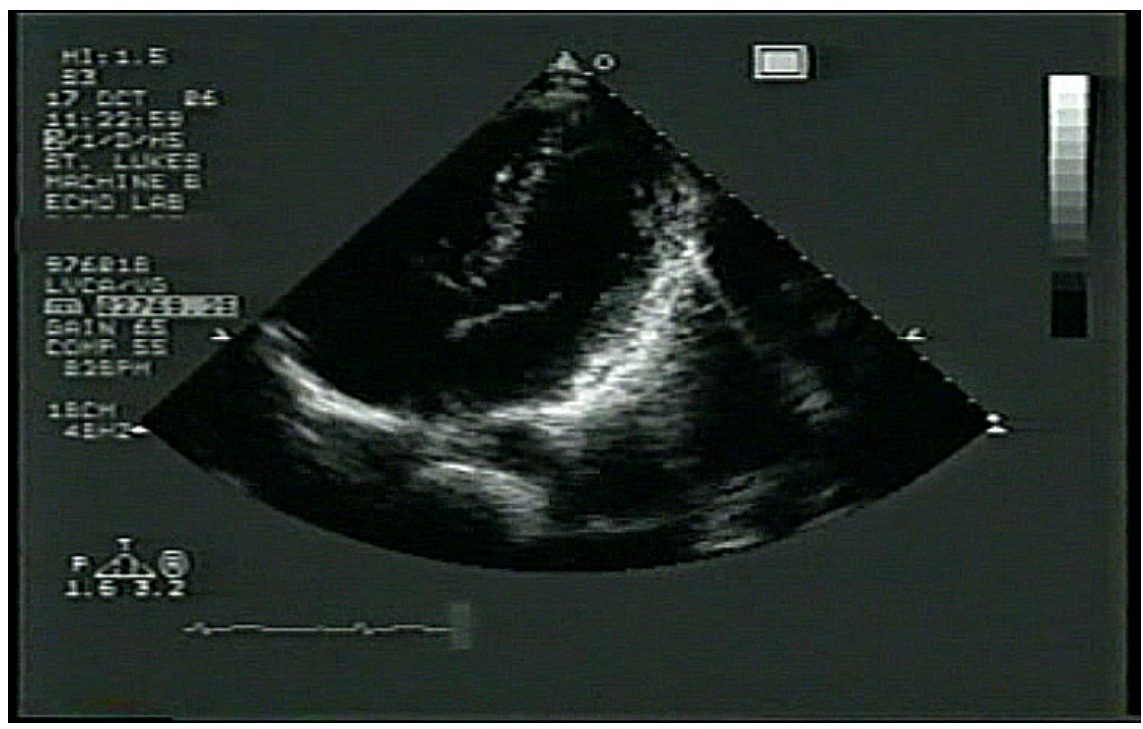

Figure 4: Echocardiogram at the end of systole.

The echocardiogram at the end of systole as shown in fig. 4 no longer shows both the apex and the mitral valve. Much of this difficulty arises because 2-D slices of 3-D images are studied here. Part of the problem can be seen from a comparison of the MRI results for the ends of both diastole and systole in fig. 5 . It can be seen that the apex at the end of systole is shifted by about $1 \frac{1 / 2}{\mathrm{~cm}}$ to the left. In order to undertake fluid calculations using existing numerical schemes it was necessary to move the end of systole result to the right while maintaining the shape.

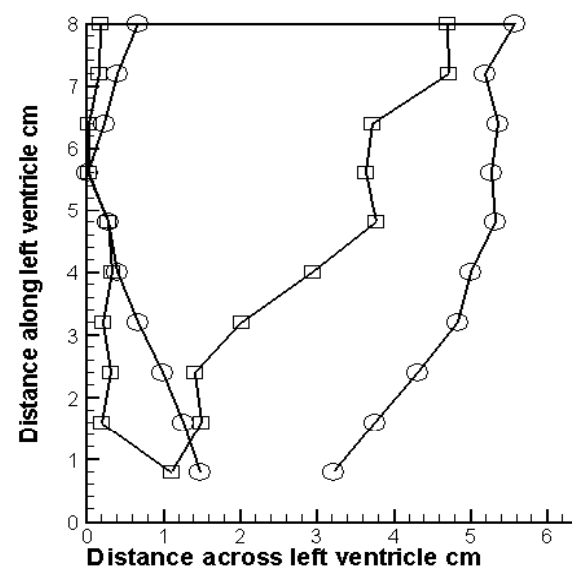

Figure 5: Ventricle shape at the end of systole $\square-\square$, end of diastole ○—○—०. 
The calculated shear stress (stress parallel to wall) approaching the end of systole is shown in fig. 6. The corresponding flow pattern is shown in fig. 7. In this as in figure 8 the velocity vector unit is 0.011 grid lengths representing 1 $\mathrm{cm} / \mathrm{sec}$. The aortic valve is simulated by a sink in the upper left corner. It can be seen that the stress developed near the apex is high. If the shape of the apex is not well defined then important details would be lost. The flow pattern at an earlier time is shown for comparison in fig. 8 .

The pressure (normal stress) difference variation is shown in fig 9. It can be seen that the maximum pressure occurs at the aortic valve. As the end of systole is approached, the flow decreases the stresses become less.

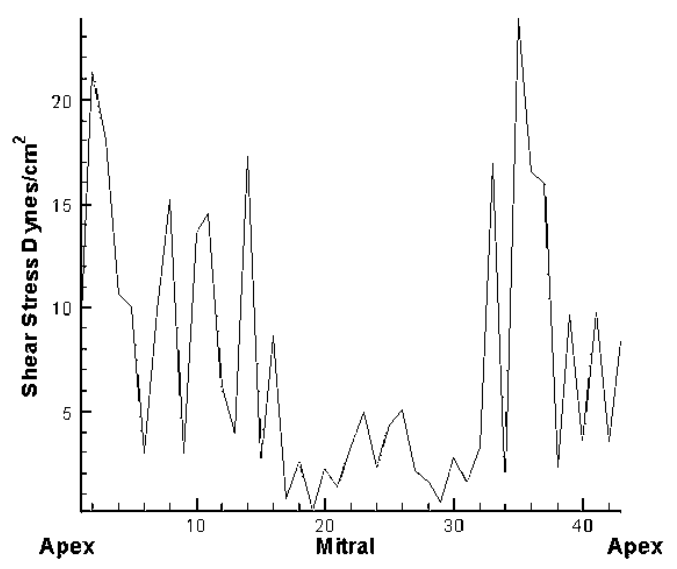

Figure 6: The variation of stress on the ventricular walls during systole.

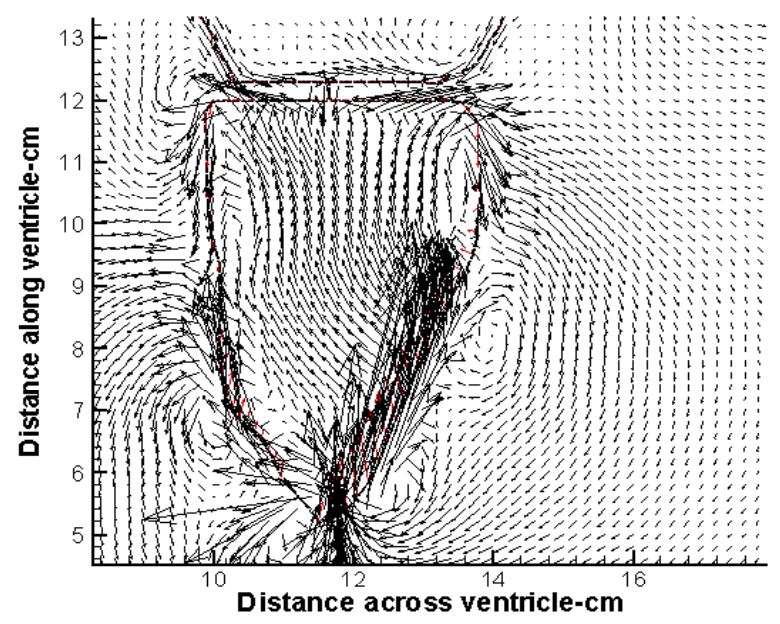

Figure 7: The flow pattern corresponding to fig. 6. 


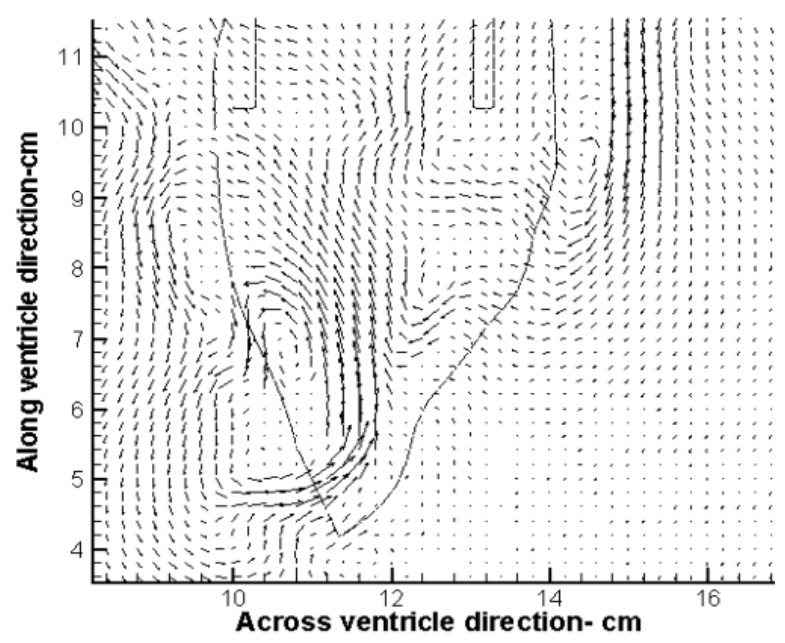

Figure 8: $\quad$ Flow pattern before mitral valve closed.

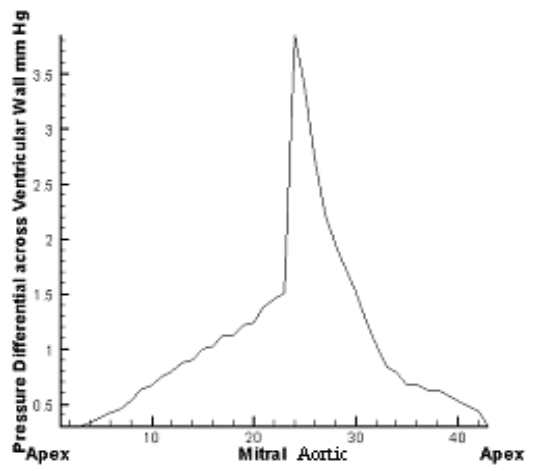

Figure 9: Variation of pressure around ventricle under conditions of fig .6.

\section{Conclusions and future work}

It has been shown that under certain conditions near the end of systole accurate depiction of the ventricle near the apex is very important. This is close to the time period when peak values of shear stress occur. In the present case this could be obtained from the available patient MRIs. However in most clinical practice it will be necessary to use echocardiogram results for the immediate future.

The penetration of Ang II is determined by the normal velocity to the surface. It can be seen that during systole that the flow is mainly parallel to the walls. However as shown in fig. 8 there are larger components of velocity normal to the wall when the mitral valve is open. 
One consideration that can only be addressed by clinical work is whether the important stress levels are the peak values or average lower level values of stress that could be higher in abnormal patients. More extensive examination of echocardiograms and MRIs of the same patient are required to determine if it is possible to determine the shape of the ventricle during the latter part of systole.

To produce more accurate results in the future, it will be necessary to alter the computational algorithms to allow for the sideways movement of the ventricle at the end of systole.

\section{References}

[1] Ruwhof C, van der Laarse A. "Mechanical stress-induced cardiac hypertrophy: mechanisms and signal transduction pathways" Cardiovascular Research 47 23-37, 2000.

[2] Sadoshima J. and Izumo S. "The cellular and molecular response of cardiac myocytes to mechanical stress" Ann. Revs of Physiology, 59,551$571,1997$.

[3] Dzau V.J., Gibbons G.H., Autocrine and paracrine mechanisms of vascular myocytes in systemic hypertension Amer. J Cardiol 60, 9911031, 1987.

[4] Saavedra JM, Benicky J, Zhou J. Mechanisms of the Anti-Ischemic Effect of Angiotensin II AT( 1 ) Receptor Antagonists in the Brain. Cell Mol Neurobiol., Nov;26(7-8):1097-1099, 2006.

[5] Macpherson, A.K., Neti, S and Macpherson, P.A. "Blood Flow induced wall stress in the left ventricle of the heart ", Advances Fluid Mechanics V, eds M. Rahman, R. Verhoeven, C.A. Brebbla, WIT press, Southampton, 321-331, 2004.

[6] Macpherson AK and Neti S, "The effect of Angiotensin II on heart blood flow and hypertension", Advances Fluid Mechanics IV, eds M. Rahman, R. Verhoeven, C.A. Brebbla, WIT press, Southampton, 1-12, 2002.

[7] Macpherson, A.K. and Neti, S. "Modelling the Effects of Angiotensin Receptor Blockers on Left Ventricle Remodelling," JRASS, 2,1,52, 2001.

[8] Macpherson, A.K. and Neti S, "A rapid procedure for initial drug evaluation," Phys. Med. Biol, June; 46(6): N139-47, 2001.

[9] Macpherson, A.K. and Neti, S. "Simulating Physiology and Methods for Therapeutic Evaluation with Emphasis on Hypertension," Current Topics in Medicinal Chemistry, No. 4, 461-471, 2004.

[10] Morgan, KT, et al "Frequent Sampling Reveals Dynamic Behavior Throughout the Transcriptome of HepG2 Cells Following Nutrient Replacement", Toxicologic Pathology, 31,448-461,2003.

[11] Macpherson, A.K. Macpherson, P.A. and Neti, S. "Bloodflow induced wall stress in the left ventricle of the heart " Modelling in Medicine and Biology VI, eds M. Ursino, C.A. Brebbla, G. Pontrelli and E. Magosso, WIT press, Southampton, 171-179, 2005. 